\title{
Introduction to the HICSS-55 Minitrack on Process Mining in Healthcare
}

\author{
Luise Pufahl \\ Technische Universitaet Berlin \\ luise.pufahl@tu-berlin.de
}

\author{
Jorge Munoz-Gama \\ Pontificia Universidad Católica de \\ Chile \\ jmun@ing.puc.cl
}

\author{
Mathias Weske \\ HPI, University of Potsdam \\ mathias.weske@hpi.de
}

This minitrack provides a forum to discuss novel ideas and techniques as well as experiences in the area of process mining in healthcare. Process mining has gained increasing attention recently in the field of information science as a set of concepts, methods, and techniques to analyze the execution of business processes. In traditional application domains of business process technology including finance and electronic commerce, process mining provides insights about how exactly business processes are performed. Since then, process mining has experienced an explosion of its use to other, novel application scenarios, including logistics, public administration, and, more prominently, healthcare. The application of process mining in healthcare is faced with several challenges due to the flexible nature of the healthcare processes and the focus on patient needs. Nevertheless, healthcare information systems provide a rich set of process-oriented data, and process mining offers the chance to provide more transparency and room for improving healthcare processes. This minitrack is aimed at discussing new ideas to apply process mining techniques successfully in the healthcare domain with researchers and practitioners.

The first edition of the minitrack attracted seven submissions, from which three regular papers were accepted. These contributions address a range of topics, such as predicting the nurse workload, uncovering complex relations in patient pathways, and coupling AI and process-oriented healthcare systems:

In the paper "An Insight to Nurse Workload: Predicting Activities in the Next Shift and Analyzing Bedside Alarms Influence" Renata Medeiros de Carvalho, Huyen Nguyen, Maikel Heetveld, and Jolanda Luime address organizational aspects of healthcare processes by investigating the role of nurses in the context of bedside alarms. This study uses event abstraction and prediction techniques to substantiate its interesting findings.

Patient treatment processes are at the center of the paper "Uncovering Complex Relations in Patient
Pathways based on Statistics: the Impact of Clinical Actions" by Imer Jan Koorn, Xixi Lu, Felix Mannhardt, Henrik Leopold, and Hajo Reijers. The authors propose a statistical approach that aims at providing insights into dependencies between activities in healthcare processes that so far have been hidden. The approach is applied to a publicly available sepsis event $\log$.

The paper "PathwAI Systems in Healthcare - a Framework for Coupling AI and Process-centered Health Information Systems" by Tim Scheplitz, Martin Burwitz, and Thure Weimann reviews the use of artificial intelligence techniques on patient treatment processes in healthcare. In particular, adaptive behavior and learning effects that are typically found in healthcare processes are used as a key ingredient to review and evaluate artificial intelligence methods and techniques.

We trust that this year's contributions will stimulate interesting discussions and will advance the research in the field of process mining and healthcare.

The organizers would like to thank all the Program Committee members for their valuable work in reviewing the papers, and the HICSS-55 organizing committee for supporting the first edition of this minitrack.

The minitrack was supported by the Process-Oriented Data Science for Healthcare Alliance (PODS4H Alliance). The goal of this international alliance is to promote the research, development, education, and understanding of process-oriented data science in healthcare. For more information about the activities and their members, visit pods4h.com/alliance. 